BULLETIN OF THE

AMERICAN MATHEMATICAL SOCIETY

Volume 77, Number 4, July 1971

\title{
TIGHT EQUIVARIANT IMMERSIONS OF SYMMETRIC SPACES
}

\author{
BY EDMUND KELLY
}

Communicated by Raoul Bott, July 6, 1970

Introduction. Let $G / K$ be a compact, irreducible symmetric space and $\mathfrak{g}=\mathfrak{f}+\mathfrak{p}$ the Lie algebra of $G$. If $\pi$ is a nontrivial real class-one representation of $G$ on $E^{N}$ with $0 \neq e, K$-fixed, then the map $\pi: G / K$ $\rightarrow E^{n}$ given by $g K \rightarrow \pi(g) e$ gives an immersion of $G / K$ into $E^{N}$. The purpose of this note is to announce the classification of such immersions with minimal absolute curvature (i.e., are tight) [1], [4].

In a slightly different vein is the problem of finding to what symmetric spaces can the work of Frankel [2] be extended. One can describe Frankel's method as "take an equivariant immersion of a homogeneous space and examine the critical manifolds for nondegenerate height functions." The present work shows that to extend Frankel's results to spaces which are not $R$-spaces, the exceptional groups for instance, will require some modification of method.

The author is indebted to Professor Sigurdur Helgason for his unfailing encouragement and many useful discussions.

Tightness. If $M$ is a compact, $n$-dimensional connected $C^{\infty}$ manifold and $\phi$ is a real $C^{\infty}$ function on $M$ with nondegenerate critical points then

$$
\begin{aligned}
\beta_{k}(\phi) & =\text { number of critical points of index } k \text { of } \phi, \\
\beta_{k}(M) & =\text { minimum }\left\{\beta_{k}(\phi) \mid \phi \text { nondegenerate }\right\}, \\
\beta(M) & =\text { minimum }\left\{\sum_{k=1}^{n} \beta_{k}(\phi) \mid \phi \text { nondegenerate }\right\} .
\end{aligned}
$$

If $f: M \rightarrow R^{N}$ is an immersion the height functions on $M$ are the functions

$$
\phi_{a}(x)=(a, f(x)) \quad \text { where } a \in R^{N} .
$$

$f$ is tight ( $k$-tight) if $\beta\left(\phi_{a}\right)=\beta(M)\left(\beta_{k}\left(\phi_{a}\right)=\beta_{k}(M)\right)$ when $\phi_{a}$ is nondegenerate.

AMS 1970 subject classifications. Primary 53C35, 53C40; Secondary 57D70, 53B25.

Key words and phrases. Tight, total absolute curvature symmetric spaces, equivariant immersion, second fundamental form.

Copyright (c) 1971, American Mathematical Society 
Symmetric $R$-spaces. Let $\&$ be a real noncompact semisimple Lie algebra with $Z \in \mathbb{R}$ such that ad $Z$ is semisimple with eigenvalues 0 , \pm 1 . There is a Cartan decomposition $\mathfrak{l}=\mathfrak{g}+\beta$ with $Z \in \beta$. If $L$ is a Lie group without center and with Lie algebra $R$ and $G$ is the subgroup of $L$ corresponding to $g$ and if $K=\{g \in G \mid \operatorname{Ad} g Z=Z\}$ then $G / K$ is symmetric, and is called a symmetric $R$-space. We have of course the imbedding

$$
G / K \rightarrow \beta \quad \text { by } g K \rightarrow \operatorname{Ad} g Z .
$$

Second fundamental form. If $f: M \rightarrow N$ is an immersion of a manifold in a Riemannian manifold and $N_{x}=M_{x} \oplus M_{x}^{1}$ is the decomposition of the tangent space at $x \in N$ under the Riemannian metric then it is convenient to regard the second fundamental form of the immersion at $x$ as the bilinear symmetric map $\alpha: M_{x} \times M_{x} \rightarrow M_{x}^{\perp}$ constructed as follows: if $X, Y \in M_{x}$, and $\tilde{X}, \tilde{Y}$ are tangential vector fields with $\tilde{X}_{x}=\tilde{X}, \tilde{Y}_{x}=Y$, then

$\alpha(X, Y)=$ normal component of $\left(\bar{\nabla}_{X} Y\right)_{x}$ where $\bar{\nabla}$ is the

Riemannian connection on $N$.

Theorem 1. Let $G / K$ be an irreducible compact symmetric space and $\mathfrak{g}=\mathfrak{f}+\mathfrak{p}$ the Lie algebra of $G$. If $\pi$ is a real class-one representation of $G$ on $E^{N}$ with $0 \neq e, K$-fixed, and $\pi$ also denotes the corresponding representation of $g$, then for the immersion $\pi: G / K \rightarrow E^{N}$ given by $g K \rightarrow \pi(g) e$

$$
\alpha(X, Y)=\pi(X) \pi(Y) e \text { for } X, Y \in \mathfrak{p} .
$$

The following theorem is an useful improvement of Theorem 4 [4].

TheOREM 2. If $f: M \rightarrow R^{N}$ is a 0 -tight immersion of a compact connected manifold then there is an open set $U \subset M$ such that the second fundamental form is an onto map at each point of $U$.

REMARK ON PROOF OF THEOREM 2. This theorem represents a change in the point of view from [4] more than anything else. Using local coordinates $\left\{x_{i}\right\}$ about the point $m$ in $M$

$$
\alpha\left(\left(\frac{\partial}{\partial x_{i}}\right)_{m},\left(\frac{\partial}{\partial x_{j}}\right)_{m}\right)=\text { normal component of } \frac{\partial^{2} f}{\partial x_{i} \partial x_{j}} \text { at } m .
$$

Now if $\phi_{a}$ is a nondegenerate height function with its maximum at $m$ then its Hessian at $m$ is the matrix

$$
\left[\left(a, \alpha\left(\left(\frac{\partial}{\partial x_{i}}\right)_{m},\left(\frac{\partial}{\partial x_{j}}\right)\right)\right)\right] .
$$


If $\alpha$ is not onto we can find $z \in M_{m}$ such that $\phi_{a+z}$ has a nondegenerate critical point of index $n$ at $m$ but its absolute maximum elsewhere. Then by standard "Morse Theory techniques" we can get a $\phi_{v}$ close to $\phi_{a+z}$ such that $\phi_{v}$ is nondegenerate.

Main result. One can reduce the classification of tight equivariant immersions of irreducible symmetric spaces to the examination of irreducible representations and we get

THeORem 3. Let $G / K$ be an irreducible compact locally symmetric space and $\pi$ an irreducible class-one representation of $G$ giving the immersion $\pi: G / K \rightarrow E^{N}$. Then the following are equivalent:

(i) $\pi$ is 0 -tight.

(ii) $G / K$ is a symmetric $R$-space and $\pi$ is one of the imbeddings (A).

(iii) $\pi$ is tight (has minimal total curvature).

REMARK ON PROOF. (ii) $\Rightarrow$ (iii) was proved in [3].

(iii) $\Rightarrow$ (i). See [4] and [5].

(i) $\Rightarrow$ (ii). The central idea in the proof is the following: Let \& $=\mathfrak{g} \oplus E^{N}$. Give $\mathfrak{R}$ the following algebraic structure:

(i) $X, Y$ in $\mathfrak{g}:[X, Y]$ as in $\mathfrak{g}$.

(ii) $X$ in $\mathfrak{g}, u$ in $E^{N}:[X, u]=-[u, X]=\pi(X) u$.

(iii) $u, v$ in $E^{N}$ then $[u, v]$ is in $\mathfrak{g}$ where

$$
-B([u, v], X)=(v, \pi(X) u) \text { for all } X \text { in } \mathfrak{g},
$$

where the inner product on the right is the Euclidean inner product on $E^{N}$ and $B$ is the Killing-form on $\mathrm{g}$. Then we have

Lemma 1. Under the assumptions of Theorem 3, Part (i), this algebraic structure makes $\mathfrak{R}=\mathfrak{g}+E^{N}$ into a Lie algebra.

Details will appear elsewhere.

Acknowledgments. These results appeared in the author's doctoral thesis written under Professor S. Helgason at Massachusetts Institute of Technology.

For a time at M.I.T., the author was supported by a Northern Ireland Ministry of Education Postgraduate Studentship.

\section{BIBLIOGRAPHY}

1. S.-S. Chern and R. K. Lashof, On the total curvature of immersed manifolds. I, Amer. J. Math. 79 (1957), 306-318; II, Michigan Math. J. 5 (1958), 5-12, MR 18, 927; MR $20 \# 4301$.

2. T. T. Frankel, Critical submanifolds of classical groups and Stiefel manifolds, 
Differential and Combinatorial Topology (A Symposium in Honor of Marston Morse), Princeton Univ. Press, Princeton, N.J., 1965, pp. 37-53. MR 33 \#4952.

3. M. Takeuchi and S. Kobayashi, Minimal imbeddings of R-spaces, J. Differential Geometry 2 (1968), 203-215. MR 39 \#366.

4. N. Kuiper, Immersions with minimal total absolute curvature, Colloq. Géométrie Différentielle Globale (Bruxelles, 1958), Centre Belge Rech. Math., Louvain, 1959, pp. 75-88. MR 23 \#A608.

5. M. Morse, The existence of polar non-degenerate functions on differentiable manifolds, Ann. of Math. (2) 71 (1960), 352-383. MR 22 \#4070.

Boston College, Chestnut Hill, Massachusetts 02167

University of New Brunswick, Fredericton, New Brunswick 